\title{
Non-Manipulable Domains for the Borda Count
}

\author{
Martin Barbie, Clemens Puppe and Attila Tasnádi
}

Department of Economics, University of Bonn

Adenauerallee 24-42, D - 53113 Bonn, Germany

Appeared in Economic Theory 27(2006), 411-430.

(c)Springer-Verlag

The original article is available at www.springerlink.com.

DOI: $10.1007 / \mathrm{s} 00199-004-0603-4$

July 2003

\begin{abstract}
We characterize the preference domains on which the Borda count satisfies Arrow's "independence of irrelevant alternatives" condition. Under a weak richness condition, these domains are obtained by fixing one preference ordering and including all its cyclic permutations ("Condorcet cycles"). We then ask on which domains the Borda count is non-manipulable. It turns out that it is non-manipulable on a broader class of domains when combined with appropriately chosen tie-breaking rules. On the other hand, we also prove that the rich domains on which the Borda count is non-manipulable for all possible tie-breaking rules are again the cyclic permutation domains.
\end{abstract}

\footnotetext{
${ }^{*}$ The third author gratefully acknowledges the financial support from the Deutsche Forschungsgemeinschaft (DFG), Graduiertenkolleg 629 at the University of Bonn.
} 


\section{Introduction}

Ever since their publication, the two most important results of social choice theory, the impossibility theorems of Arrow and Gibbard-Satterthwaite, have led to a steady search for possibility results on restricted domains (see Gaertner (2002) for a recent overview). The usual approach is to fix an appropriate set of admissible preferences, and to investigate which social welfare functions satisfy Arrow's conditions, respectively which social choice functions are non-manipulable, on that preference domain. Classic examples of this approach are Black (1958) and Moulin (1980) who consider the domain of single-peaked preferences 1

A somewhat different view on the question has been developed by Dasgupta and Maskin (2000) based on Maskin (1995). These authors consider specific preference aggregation rules such as majority rule, plurality rule or the Borda count, and ask on what domains these rules satisfy desirable conditions in the spirit of Arrow's conditions. The present paper follows this approach. Specifically, we restrict our attention here to the Borda count and, slightly more generally, to scoring methods (cf. Moulin (1988)). We characterize the preference domains on which scoring methods satisfy Arrow's conditions ("Arrovian domains"). In contrast to Dasgupta and Maskin (2000), we impose the original independence of irrelevant alternatives condition, not their stronger neutrality condition. By consequence, the Arrovian domains for the Borda count determined here encompass the domains that satisfy Dasgupta and Maskin's characterizing condition of "quasi-agreement." Our analysis also shows that all Arrovian domains for the Borda count that are minimally "rich" in the sense that any social alternative is on top of at least one preference ordering, are obtained by fixing one preference ordering over the alternatives and including all its cyclic permutations. Remarkably, these are precisely the configurations of preferences that give rise to the Condorcet paradox. The rich domains on which the Borda count "works well" thus turn out to be exactly the problematic domains for majority voting ${ }^{2}$

We then consider the question on which domains the Borda count is strategy-proof. Since the Borda count does in general not select one single social alternative, we have to consider tie-breaking rules here. It turns out that the Borda count is strategy-proof with any given tie-breaking rule on all Arrovian domains. The converse is not true, however. We show by example that there exist rich domains on which the Borda count violates the independence of irrelevant alternatives condition but is nevertheless strategy-proof when combined with some suitable tie-breaking rule. On the other hand, under the richness condition, strategy-proofness of the Borda count with all tie-breaking rules yields again exactly the Arrovian domains (one fixed preference ordering together with all its cyclic permutations).

Our analysis confirms the general view of the literature that the Borda count is highly vulnerable to strategic manipulation ${ }^{3}$ This intuition is made precise here in

\footnotetext{
${ }^{1}$ See also Barberá, Sonnenschein and Zhou (1991) and Barberá, Gul and Stacchetti (1993) for multidimensional extensions, and Nehring and Puppe (2003a,b) for a unifying approach to impossibility and possibility results based on "generalized single-peaked preferences" in the context of strategyproofness.

${ }^{2}$ The apparent conflict of this conclusion with Dasgupta and Maskin's (2000) robustness result for majority voting is due to the fact that we do not impose neutrality here.

${ }^{3}$ Recently, there have been different approaches to measuring the degree of "vulnerability" of voting procedures to strategic manipulation, see Aleskerov and Kurbanov (1999) and Smith (1999), among others. The most relevant study in our context is Favardin, Leppelley and Serais (2002) who character-
} 
two ways. First, for any preference ordering, there is only one rich domain that contains the given preference ordering and that renders the Borda count non-manipulable. By contrast, for other choice rules there are frequently many different rich and nonmanipulable domains that contain a given preference ordering; for instance, there are many rich single-peaked domains that contain a given single-peaked preference ordering. Secondly, any fixed rich domain on which the Borda count is non-manipulable is as small as it could possibly be, since it contains just as many orderings as there are social states. Again, this strong restriction does not apply to single-peaked domains, for instance. Thus, the overall conclusion from our analysis is that the Borda count fares poorly in terms of strategic manipulation, in the sense that there are very few non-manipulable domains all of which are, moreover, very small.

The plan of the paper is as follows. In Section 2 we introduce our basic notation and definitions. Section 3 provides the characterization of the Arrovian domains for general scoring methods. In Section 4, we consider the most interesting special case of the Borda count. For this case, we provide an alternative global characterization which in particular yields the cyclic permutation structure of all rich Arrovian domains. In Section 5, we then investigate the structure of non-manipulable domains. While some proofs are included in the main text, the more technical ones are collected in an appendix.

\section{Basic Notation and Definitions}

\subsection{Social Welfare Functionals and the Arrow Conditions}

Let $X$ be a finite universe of social states or social alternatives. By $\mathcal{P}_{X}$, we denote the set of all linear orderings (irreflexive, transitive and total binary relations) on $X$, and by $\mathcal{P} \subseteq \mathcal{P}_{X}$ a generic subdomain of the unrestricted domain $\mathcal{P}_{X}$. Moreover, denote by $\mathcal{R}$ the set of all weak orderings (reflexive, transitive and complete binary relations).

Definition (Social welfare functional) A mapping $F: \bigcup_{n=1}^{\infty} \mathcal{P}^{n} \rightarrow \mathcal{R}$ that assigns a social preference ordering $F\left(\succ_{1}, \ldots, \succ_{n}\right) \in \mathcal{R}$ to each $n$-tuple of linear orderings and all $n$ is called a social welfare functional ( $S W F$ ).

Thus, we do allow for non-trivial indifferences on the social level but not on the individual level. Note also that we require a SWF to be defined for societies with any finite number of agents. For some of our results this will be important. Alternatively, we could have assumed a continuum of agents as e.g. in Dasgupta and Maskin (2000).

A SWF $F$ satisfies the Pareto rule on $\mathcal{P}$ if, for all $x, y \in X$, all $\succ_{i} \in \mathcal{P}$ and all $n$,

$$
\left[x \succ_{i} y \text { for all } i=1, \ldots, n\right] \Rightarrow x \succ y,
$$

where $\succ$ is the strict part of the social preference relation $\succeq=F\left(\succ_{1}, \ldots, \succ_{n}\right)$.

A SWF $F$ is called non-dictatorial on $\mathcal{P}$ if, either $\# \mathcal{P}=1$ or, for all $n \geq 2$ and all $i=1, \ldots, n$, there exist $x, y \in X$ and $\succ_{i} \in \mathcal{P}$ such that $x \succ_{i} y$ and $y \succeq x$, where $\succeq=F\left(\succ_{1}, \ldots, \succ_{n}\right)$.

ize (for the case of three alternatives) the preference profiles at which the Borda count is manipulable. Their conclusion is that the Borda count is significantly more vulnerable than, say, the Copeland method. Note that, in contrast to this literature, our aim is not to determine the relative frequency of possible manipulation on an unrestricted domain, but to characterize the restricted domains on which manipulation can never occur. 
A SWF $F$ satisfies independence of irrelevant alternatives (IIA) on $\mathcal{P}$ if, for all $x, y \in X$, all $n$ and all $\succ_{i}, \succ_{i}^{\prime} \in \mathcal{P}$,

$$
\left.\left[\left.\succ_{i}\right|_{\{x, y\}}=\left.\succ_{i}^{\prime}\right|_{\{x, y\}} \text { for all } i=1, \ldots, n\right] \Rightarrow \succeq\right|_{\{x, y\}}=\left.\succeq^{\prime}\right|_{\{x, y\}},
$$

where $\succeq=F\left(\succ_{1}, \ldots, \succ_{n}\right), \succeq^{\prime}=F\left(\succ_{1}^{\prime}, \ldots, \succ_{n}^{\prime}\right)$, and $\left.\succ\right|_{\{x, y\}}$ denotes the restriction of the binary relation $\succ$ to the pair $\{x, y\}$.

Definition (Scoring method) Let $q$ be the cardinality of $X$, and let $s:\{1, \ldots, q\} \rightarrow \mathbf{R}$ satisfy $s(1) \geq s(2) \geq \ldots \geq s(q)$ and $s(1)>s(q)$. Moreover, let $r k[x, \succ]$ denote the rank of alternative $x$ in the ordering $\succ$ (i.e. $r k[x, \succ]=1$ if $x$ is the top alternative in the ranking $\succ, r k[x, \succ]=2$ if $x$ is second-best, and so on). A SWF is a scoring method if for some function $s$, all $x, y \in X$, all $n$ and all $\succ_{i}, i=1, \ldots, n$,

$$
x \succeq y \Leftrightarrow \sum_{i=1}^{n} s\left(r k\left[x, \succ_{i}\right]\right) \geq \sum_{i=1}^{n} s\left(r k\left[y, \succ_{i}\right]\right),
$$

where $\succeq$ is the social preference corresponding to $\left(\succ_{1}, \ldots, \succ_{n}\right)$. The scoring method corresponding to the function $s:\{1, \ldots, q\} \rightarrow \mathbf{R}$ will be denoted by $F^{s}$. A scoring method is called proper if $s$ is strictly decreasing.

Definition (Borda count) The Borda count (or "rank-order voting rule"), denoted by $F^{B}$, is the proper scoring method corresponding to the function $s(k)=q+1-k$ for $k=1, \ldots, q$.

Clearly, all scoring methods are non-dictatorial; moreover, any proper scoring method satisfies the Pareto rule. On the other hand, scoring methods do not generally satisfy the IIA condition. A characterization of the domains on which scoring methods satisfy this condition will be provided in Section 3 below.

\subsection{Social Choice Functions and Non-Manipulability}

Definition (Social choice function) A mapping $f: \bigcup_{n=1}^{\infty} \mathcal{P}^{n} \rightarrow X$ that assigns a social alternative to each $n$-tuple of linear orderings and all $n$ is called a social choice function $(S C F)$.

A SCF $f$ satisfies unanimity on $\mathcal{P}$ if, for all $x \in X$, all $\succ_{i} \in \mathcal{P}$ and all $n$,

$$
\left[r k\left[x, \succ_{i}\right]=1 \text { for all } i=1, \ldots, n\right] \Rightarrow x=f\left(\succ_{1}, \ldots, \succ_{n}\right) .
$$

A SCF $f$ is called non-manipulable, or strategy-proof on $\mathcal{P}$ if for all $n$, all $\succ_{i}, \succ_{i}^{\prime} \in \mathcal{P}$ and all $\succ_{-i} \in \mathcal{P}^{n-1}$,

$$
f\left(\succ_{i}, \succ_{-i}\right) \succeq_{i} f\left(\succ_{i}^{\prime}, \succ_{-i}\right)
$$

Example (Borda count with tie-breaking rule) For our purposes, a tie-breaking rule is simply a linear ordering $\tau$ on $X$. Given a tie-breaking rule $\tau$, any SWF $F$ uniquely defines a SCF $f$ by associating to each preference profile $\left(\succ_{1}, \ldots, \succ_{n}\right)$ the $\tau$ best element of $F\left(\succ_{1}, \ldots, \succ_{n}\right) \subseteq X$. Below we will specifically consider the Borda count $F^{B}$ together with a tie-breaking rule $\tau$; the resulting SCF will be denoted by $f_{\tau}^{B}$. Note that $f_{\tau}^{B}$ satisfies unanimity.

Obviously, sufficiently "small" domains can give rise to strategy-proofness in a trivial 
way. For instance, any SCF is vacuously strategy-proof on any domain consisting of one single preference ordering. We will therefore be often interested in domains that are "rich" in the sense that any alternative is on top of some preference ordering 4

Definition (Rich domain) A domain $\mathcal{P}$ is called rich if for any $x \in X$ there exists $\succ \in \mathcal{P}$ such that $r k[x, \succ]=1$.

\section{Arrovian Domains for Scoring Methods}

It is well-known that scoring methods violate the IIA condition on the unrestricted domain $\mathcal{P}_{X}$. However, scoring methods may well satisfy this condition on restricted domains. We will say that $\mathcal{P}$ is an Arrovian domain for the SCW $F$ if $F$ is nondictatorial and satisfies the Pareto rule as well as IIA on $\mathcal{P}$.

Definition (Equal score difference) A domain $\mathcal{P}$ satisfies the equal score difference condition with respect to $s$ if, for all $x, y \in X$, either all orderings in $\mathcal{P}$ agree on $\{x, y\}$, or if not, then

$$
s(r k[x, \succ])-s(r k[y, \succ])=s\left(r k\left[x, \succ^{\prime}\right]\right)-s\left(r k\left[y, \succ^{\prime}\right]\right)
$$

for all $\succ, \succ^{\prime} \in \mathcal{P}$ such that $\left.\succ\right|_{\{x, y\}}=\left.\succ^{\prime}\right|_{\{x, y\}}$.

Note that for the Borda count the latter condition reduces to

$$
r k[x, \succ]-r k[y, \succ]=r k\left[x, \succ^{\prime}\right]-r k\left[y, \succ^{\prime}\right]
$$

for all $\succ, \succ^{\prime} \in \mathcal{P}$ such that $\left.\succ\right|_{\{x, y\}}=\left.\succ^{\prime}\right|_{\{x, y\}}$, which we will also refer to as the equal rank difference condition.

Theorem 1 A domain is Arrovian for the proper scoring method $F^{s}$ if and only if it satisfies the equal score difference condition with respect to $s$.

Proof Clearly, any scoring method is non-dictatorial and satisfies the Pareto rule on any domain. Let $\mathcal{P}$ satisfy the equal score difference condition. In order to verify IIA consider any $x, y \in X$ and $\succ_{i} \in \mathcal{P}$ for $i=1, \ldots, n$. Suppose that $x \succeq y$, where $\succeq=F^{s}\left(\succ_{1}, \ldots, \succ_{n}\right)$, i.e. suppose that

$$
\sum_{i=1}^{n}\left[s\left(r k\left[x, \succ_{i}\right]\right)-s\left(r k\left[y, \succ_{i}\right]\right)\right] \geq 0 .
$$

If all orderings in $\mathcal{P}$ agree on $\{x, y\}$, we must in fact have $x \succ y$, and this relative ranking of $x$ and $y$ holds for the social preference corresponding to any profile. Thus, assume that not all orderings in $\mathcal{P}$ agree on the pair $\{x, y\}$. Then, by the equal score difference condition, the inequality (3.1) is preserved when any voter $i$ 's ordering $\succ_{i} \in \mathcal{P}$ is replaced by an ordering $\succ_{i}^{\prime} \in \mathcal{P}$ that agrees with $\succ_{i}$ on $\{x, y\}$. This shows that $F^{s}$ satisfies IIA on $\mathcal{P}$.

Conversely, suppose that the domain $\mathcal{P}$ does not satisfy the equal score difference condition. Then, there exist $x, y \in X$ and three orderings $\succ, \succ^{\prime}, \succ^{\prime \prime} \in \mathcal{P}$ such that

$$
l:=s\left(r k\left[y, \succ^{\prime \prime}\right]\right)-s\left(r k\left[x, \succ^{\prime \prime}\right]\right)>0
$$

\footnotetext{
${ }^{4}$ This condition is often imposed in the literature. It is much weaker than the richness condition used in Nehring and Puppe (2003a).
} 
and

$$
s(r k[x, \succ])-s(r k[y, \succ])=: m>m^{\prime}:=s\left(r k\left[x, \succ^{\prime}\right]\right)-s\left(r k\left[y, \succ^{\prime}\right]\right)>0 .
$$

Choose $n_{1}$ and $n_{2}$ such that

$$
\frac{l}{m^{\prime}}>\frac{n_{1}}{n_{2}}>\frac{l}{m}
$$

and consider the following two profiles of $n_{1}+n_{2}$ individual preferences. In the first profile, denoted by $\Pi=\left(\succ, \ldots, \succ, \succ^{\prime \prime}, \ldots \succ^{\prime \prime}\right)$, the first $n_{1}$ voters have the preference $\succ$ and the remaining $n_{2}$ voters have the preference $\succ^{\prime \prime}$; in the second profile, denoted by $\Pi^{\prime}=\left(\succ^{\prime}, \ldots, \succ^{\prime}, \succ^{\prime \prime}, \ldots, \succ^{\prime \prime}\right)$, the first $n_{1}$ voters have the preference $\succ^{\prime}$, and the remaining $n_{2}$ voters have the preference $\succ^{\prime \prime}$. By construction, $x$ is ranked strictly above $y$ in the social ranking $F^{s}(\Pi)$ corresponding to the first profile, while $y$ is strictly above $x$ in the social ranking $F^{s}\left(\Pi^{\prime}\right)$ corresponding to the second profile. This yields the desired violation of IIA and completes the proof.

\section{A Special Case: The Borda Count}

The restrictiveness of the equal score difference condition depends on the scoring rule. For instance, suppose that $X=\{x, y, z\}$ and consider any scoring method $s$ that does not coincide with the Borda count, i.e. $s(2)-s(1) \neq s(3)-s(2)$. It is easily seen that any domain that satisfies the equal score difference condition with respect to such $s$ can consist of at most two preference orderings on $X$. More generally, one can show that, for arbitrary $X$, no scoring method different from the Borda count can satisfy the equal score difference condition on any rich domain. On the other hand, for the Borda count there are rich domains satisfying the corresponding (equal rank difference) condition. In the following, we will provide a "global" characterization of all such domains. Before we do so, we briefly want to compare our equal rank difference condition to Dasgupta and Maskin's (2000) condition of "quasi-agreement." That condition requires that any triple $\{x, y, z\}$ admit one member, say $x$, such that all orderings in the domain agree on either (i) $x$ being the best element among the three, or (ii) $x$ being the middle element, or (iii) $x$ being the worst element among the triple. Dasgupta and Maskin (2000) show that the property of quasi-agreement characterizes the domains on which the Borda count satisfies an appropriate neutrality condition stronger than Arrow's independence of irrelevant alternatives considered here. By consequence, quasi-agreement is more restrictive than the equal rank difference condition. This can be directly verified by contraposition, as follows. Suppose that a domain violates the equal rank difference condition, i.e. there exist three orderings $\succ_{1}, \succ_{2}$ and $\succ_{3}$ such that

$$
r k\left[y, \succ_{1}\right]-r k\left[x, \succ_{1}\right]>r k\left[y, \succ_{2}\right]-r k\left[x, \succ_{2}\right]>0
$$

and $r k\left[y, \succ_{3}\right]-r k\left[x, \succ_{3}\right]<0$. By (4.1), there exists a third alternative $z$ such that $x \succ_{1} z \succ_{1} y$ but not $\left(x \succ_{2} z \succ_{2} y\right)$, in which case the three orderings violate quasiagreement on the triple $\{x, y, z\}$.

Equal rank difference as well as quasi-agreement are "local" conditions; the former imposes restrictions on any pair, the latter on any triple. It is therefore not evident how these conditions are reflected in the "global" structure of the corresponding domains. We now provide an alternative characterization of equal rank difference domains that 
makes this global structure explicit. An ordering $\succ^{\prime}$ is called a cyclic permutation of $\succ$ if $\succ^{\prime}$ can be obtained from $\succ$ by sequentially shifting the bottom element to the top while leaving the order between all other alternatives unchanged. Thus, for instance, the cyclic permutations of the ordering abcd are dabc, cdab and bcda. The set of all cyclic permutations of a fixed ordering $\succ$ is denoted by $\mathcal{Z}(\succ)$. Say that a domain $\mathcal{P}$ is hierarchically cyclic if there exists a partition $\left\{X_{1}, \ldots, X_{r}\right\}$ of $X$ such that for all $\succ \in \mathcal{P}$ and all $i \in\{1, \ldots, r\}$,

(i) $x \succ y$ whenever $x \in X_{i}, y \in X_{j}$ and $j>i$, and

(ii) $\left\{\left.\succ^{\prime}\right|_{X_{i}}: \succ^{\prime} \in \mathcal{P}\right\} \subseteq \mathcal{Z}\left(\left.\succ\right|_{X_{i}}\right)$ or $\#\left\{\left.\succ^{\prime}\right|_{X_{i}}: \succ^{\prime} \in \mathcal{P}\right\} \leq 2$

Thus, a domain is hierarchically cyclic if the universe of alternatives can be partioned in such a way that (i) the partition elements themselves are ordered unambiguously and identically by all orderings, and (ii) within each partition element $X_{i}$, the restrictions to $X_{i}$ give rise to at most two different orderings on $X_{i}$, or they are cyclic permutations of each other. The following table shows a typical domain satisfying this condition.

Table 1: A hierarchically cyclic domain

\begin{tabular}{ccccc}
$\succ_{1}$ & $\succ_{2}$ & $\succ_{3}$ & $\succ_{4}$ & $\succ_{5}$ \\
\hline$x_{1}$ & $x_{2}$ & $x_{4}$ & $x_{2}$ & $x_{4}$ \\
$x_{2}$ & $x_{3}$ & $x_{1}$ & $x_{3}$ & $x_{1}$ \\
$x_{3}$ & $x_{4}$ & $x_{2}$ & $x_{4}$ & $x_{2}$ \\
$x_{4}$ & $x_{1}$ & $x_{3}$ & $x_{1}$ & $x_{3}$ \\
$y_{1}$ & $y_{1}$ & $y_{3}$ & $y_{3}$ & $y_{1}$ \\
$y_{2}$ & $y_{2}$ & $y_{2}$ & $y_{2}$ & $y_{2}$ \\
$y_{3}$ & $y_{3}$ & $y_{1}$ & $y_{1}$ & $y_{3}$ \\
$u$ & $u$ & $u$ & $u$ & $u$ \\
$z_{1}$ & $z_{2}$ & $z_{3}$ & $z_{1}$ & $z_{2}$ \\
$z_{2}$ & $z_{3}$ & $z_{1}$ & $z_{2}$ & $z_{3}$ \\
$z_{3}$ & $z_{1}$ & $z_{2}$ & $z_{3}$ & $z_{1}$
\end{tabular}

In the example shown in Table 1 , the partition from the definition of a hierarchically cyclic domain is given by $X_{1}=\left\{x_{1}, x_{2}, x_{3}, x_{4}\right\}, X_{2}=\left\{y_{1}, y_{2}, y_{3}\right\}, X_{3}=\{u\}$ and $X_{4}=$ $\left\{z_{1}, z_{2}, z_{3}\right\}$. Note that the preferences are cyclic permutations of one fixed ordering on $X_{1}$ and $X_{4}$. The two different restrictions on $X_{2}$ are not cyclic permutations of each other; nevertheless, the domain satisfies the defining condition since $\# X_{2} \leq 2$.

Proposition 1 A domain $\mathcal{P}$ satisfies the equal rank difference condition if and only if it is hierarchically cyclic.

It is easily verified that any hierarchically cyclic domain satisfies the equal rank difference condition. The more difficult proof of the converse statement is deferred to the appendix. As an immediate corollary of Proposition 1 and Theorem 1, we obtain the following result showing that all rich Arrovian domains for the Borda count are obtained by fixing one preference ordering and including all its cyclic permutations; such domains will henceforth be referred to as cyclic permutation domains. Note that the cyclic permutation domains on three alternatives are precisely the "Condorcet cycles." 
Theorem 2 For any linear ordering $\succ$, there is exactly one rich Arrovian domain for the Borda count that contains $\succ$, namely the cyclic permutation domain $\mathcal{Z}(\succ)$.

\section{Non-Manipulable Domains}

We now want to ask on what domains the Borda count with tie-breaking rule is nonmanipulable. The following result shows that the equal score/rank difference condition is sufficient for non-manipulability.

Proposition 2 Suppose that the domain $\mathcal{P}$ satisfies the equal score difference condition. Then, any scoring method with any tie-breaking rule is strategy-proof on $\mathcal{P}$.

Proof Take any preference profile $\left(\succ_{1}, \ldots, \succ_{n}\right)$, and suppose that $x$ is the chosen alternative. Consider any alternative $y$ that voter $i$ prefers to $x$. Since $y$ was not chosen there must exist another voter $j$ such that $x \succ_{j} y$. By the equal score difference condition, any preference that favours $y$ over $x$ must display the same score difference between these alternatives as $\succ_{i}$. In particular, voter $i$ cannot change the difference in total scores of $y$ relative to $x$ by reporting a preference that favours $y$ over $x$. Since $y$ is arbitrary this shows that voter $i$ cannot successfully manipulate.

We now turn to the question of the necessary conditions for non-manipulability. This is a more difficult problem, and we will concentrate on the most interesting case of the Borda count. As already noted, if many conceivable preference orderings are excluded, strategy-proofness can result simply from the lack of misrepresentation possibilities. We will thus focus in the following on rich domains. Recall that the rich domains satisfying the equal rank difference condition are the cyclic permutation domains. First, we show by example that the Borda count may be non-manipulable also on domains that do not have the form of cyclic permutation domains, provided the tie-breaking rule is appropriately chosen.

Example (Non-manipulability without equal rank difference) Consider on the universe $X=\{a, b, c, d\}$ the domain $\left\{\succ_{I}, \succ_{I I}, \succ_{I I I}, \succ_{I V}\right\}$, where $a \succ_{I} b \succ_{I} c \succ_{I} d$, $b \succ_{I I} a \succ_{I I} d \succ_{I I} c, c \succ_{I I I} d \succ_{I I I} a \succ_{I I I} b$ and $d \succ_{I V} c \succ_{I V} b \succ_{I V} a$. Clearly, this domain is rich and not a cyclic permutation domain. Observe that the equal rank difference condition is only violated by the two pairs $(a, d)$ and $(b, c)$. Hence, manipulation is only possible between alternatives $a$ and $d$, or $b$ and $c$, respectively. In particular, one can easily check that a voter of type $I, I I, I I I$ or $I V$ can potentially benefit only by reporting type $I I, I, I V$ or $I I I$, respectively. Note that for any manipulation of this kind, a voter can increase the total score difference only of two alternatives simultaneously over the other two alternatives; moreover, any such change in the score difference is by exactly two units. This property makes the domain rather special.

We will now show that the Borda count is non-manipulable when combined with the tie-breaking rule $a \tau b \tau c \tau d$. Suppose that a profile with $n$ voters consists of $k, l, m$ and $p$ preferences of types $\succ_{I}, \succ_{I I}, \succ_{I I I}$ and $\succ_{I V}$, respectively. Then, we have

$$
\sum_{i=1}^{n} r k\left[a, \succ_{i}\right]+r k\left[d, \succ_{i}\right]=\sum_{i=1}^{n} r k\left[b, \succ_{i}\right]+r k\left[c, \succ_{i}\right]=5(k+l+m+p) .
$$

It follows from (5.1) that, if there is to be room for manipulation at all, the total scores of all four alternatives have to be close to each other. Consider the case in which $a$ was 
chosen by $f_{\tau}^{B}$; the other cases can be treated analogously. If $a$ was chosen, then only a voter of type $I I I$ might potentially benefit from manipulating (by misreporting to be of type $I V$ ). By the above observations and by the form of the tie-breaking rule, alternative $d$ could "overtake" $a$ only if before both received the same total score, or if $a$ led only by one unit. In the first case, all four alternatives received the same total score by (5.1), while in the latter case $b$ 's total score was greater or equal to the total score of $d$, again by (5.1). Hence, misreporting type $I V$ either does not change the outcome, or makes $b$ the winner, which is not beneficial to a type $I I I$ voter.

The example shows that on rich domains the equal rank difference condition is not necessary for non-manipulability of the Borda count together with a fixed tie-breaking rule. However, if we require non-manipulability of the Borda count when combined with any tie-breaking rule, the equal rank difference condition re-emerges, as shown by the following result.

Theorem 3 Suppose that the Borda count is non-manipulable on the rich domain $\mathcal{P}$ for all tie-breaking rules $\tau$. Then, $\mathcal{P}$ satisfies the equal rank difference condition, i.e. $\mathcal{P}$ is a cyclic permutation domain.

The proof of Theorem 3 is provided in the appendix. The following example shows that the richness assumption in Theorem 3 is needed. Consider on $X=\{a, b, c, d\}$ the domain consisting of the three preference orderings $a b c d, d a b c$ and $d a c b$. This domain violates the equal rank difference condition (in fact even any equal score difference condition). But the Borda count is non-manipulable with any tie-breaking rule. Indeed, alternatives $b$ and $c$ can never win, while there are obviously no manipulation possibilities between alternatives $a$ and $d$. 


\section{Appendix: Remaining proofs}

For the proof of Proposition 1, we need the following notation. For any $1 \leq i \leq j \leq$ $q=\# X$ let $\left.\succ\right|_{[i, j]}$ be the restriction of $\succ$ ranging from the $i$ th position to the $j$ th position of $\succ$, i.e., $\left.\succ\right|_{[i, j]}=\left.\succ\right|_{\left\{x_{i}, x_{i+1}, \ldots, x_{j}\right\}}$ where $x_{1} \succ \ldots \succ x_{i} \succ \ldots \succ x_{j} \succ \ldots \succ x_{q}$. In addition, for any $1 \leq i \leq j \leq q$, we define $\mathcal{P}_{[i, j]}:=\left\{\left.\succ\right|_{[i, j]}: \succ \in \mathcal{P}\right\}$. Furthermore, for any linear ordering $\succ$ on $X^{\prime} \subseteq X$ we shall denote by $T_{i}(\succ)$ the set of the top $i$ alternatives of $\succ$, i.e., $T_{i}(\succ)=\left\{x \in X^{\prime}: r k[x, \succ] \leq i\right\}$.

Proof of Proposition 1 It is straightforward to check that a hierarchically cyclic domain satifies ERD. Hence, we have to prove the converse statement.

Step 1: We construct recursively a partition of $X$. Let $i_{0}:=0$. To obtain the first partition element $X_{1}$, we determine the smallest integer $i \in\left\{i_{0}+1, \ldots, q\right\}$ satisfying

$$
\forall x \in X, \forall \succ, \succ^{\prime} \in \mathcal{P}: i_{0}<r k[x, \succ] \leq i \Leftrightarrow i_{0}<r k\left[x, \succ^{\prime}\right] \leq i .
$$

Clearly, at least $q$ satisfies A.1 and therefore there exists a smallest $i$, denoted by $i_{1}$, satisfying A.1. Set $X_{1}:=\left\{x \in X: i_{0}<r k[x, \succ] \leq i_{1}\right\}$ for some $\succ \in \mathcal{P}$. If $X_{1}=X$, then we are finished and the partition consists only of the single set $X_{1}$. If $X_{1} \neq X$, then we proceed inductively to obtain $i_{2}$ and $X_{2}$ from A.1. Repeating this procedure, we get the desired partition $X_{1}, \ldots, X_{r}$.

In the following, we only have to consider those sets $X_{j}$ for which

$$
\# \mathcal{P}_{\left[i_{j-1}+1, i_{j}\right]}=\#\left\{\left.\succ^{\prime}\right|_{X_{j}}: \succ^{\prime} \in \mathcal{P}\right\}>2 .
$$

Pick an arbitrary set $X_{j}$ satisfying $\mathrm{A} .2$, and set $\mathcal{P}_{j}:=\mathcal{P}_{\left[i_{j-1}+1, i_{j}\right]}=\left\{\succ_{1}, \ldots, \succ_{n_{j}}\right\}$ and $q_{j}:=i_{j}-i_{j-1}$. Clearly, $q_{j} \geq 3$ because of A.2.

Step 2: First, we establish that $\mathcal{P}_{j}$ contains three preference relations with different top alternatives. Obviously, not all preferences can have the same top alternative, since this would be in contradiction with the construction of $X_{j}$. Thus, suppose that the preferences in $\mathcal{P}_{j}$ have two different top alternatives. Without loss of generality we can assume that the first $p \in\left\{2, \ldots, n_{j}-1\right\}$ preferences have $a \in X_{j}$ as their top alternative, while the remaining preferences have another alternative $b \in X_{j} \backslash\{a\}$ as their top alternative. Define

$$
Y:=\left\{x \in X_{j}: \forall k, l \in\{1, \ldots, p\}, r k\left[x, \succ_{k}\right]=r k\left[x, \succ_{l}\right]\right\} .
$$

Clearly, $a \in Y$. Moreover, we must also have $r k\left[b, \succ_{k}\right]=r k\left[b, \succ_{l}\right]$ for all $k, l \in$ $\{1, \ldots, p\}$ by ERD, hence $b \in Y$. Let

$$
\begin{aligned}
J & :=\left\{k \in\left\{1, \ldots, q_{j}\right\}: \exists y \in Y, k=r k\left[y, \succ_{1}\right]\right\} \text { and } \\
i^{*} & :=\max \left\{k \in\left\{1, \ldots, q_{j}\right\}:\{1, \ldots, k\} \subseteq J\right\} .
\end{aligned}
$$

Observe that $i^{*}$ is well defined, since $\{1\} \subseteq J$.

Clearly, if $i^{*}=q_{j}$, then we have $\succ_{1}=\ldots=\succ_{p}$, which cannot be the case, since the preferences $\succ_{1}, \ldots, \succ_{p}$ are distinct. Hence, we may assume that $i^{*}<q_{j}$. ERD implies that any alternative $z \in X_{j} \backslash Y$ must be ranked below the $i^{*}$ th position by any preference relation having $b$ on top, since $z$ changes its rank difference to all alternatives in $T_{i^{*}}\left(\succ_{1}\right)=\ldots=T_{i^{*}}\left(\succ_{p}\right)$. Formally,

$$
\forall z \in X_{j} \backslash Y, \forall l \in\left\{p+1, \ldots, n_{j}\right\}: r k\left[z, \succ_{l}\right]>i^{*} .
$$


Suppose now that none of the alternatives in $Y$ are ranked lower than $i^{*}$ (i.e. suppose that $\left.\left\{1, \ldots, i^{*}\right\}=J\right)$. Then, we obtain $T_{i^{*}}\left(\succ_{1}\right)=\ldots=T_{i^{*}}\left(\succ_{n_{j}}\right)$ by A.4, which contradicts the construction of $X_{j}$.

Thus, there exists an alternative $y \in Y$ with $r k\left[y, \succ_{1}\right]>i^{*}+1$ (i.e. $\left\{1, \ldots, i^{*}\right\} \neq J$ ). In this case, we will show that

$$
\forall y \in Y, \forall l \in\left\{p+1, \ldots, n_{j}\right\}: r k\left[y, \succ_{1}\right]>i^{*}+1 \Rightarrow r k\left[y, \succ_{l}\right]>i^{*}[5
$$

Indeed, suppose that this is not the case, i.e. suppose that $y \in Y$ is such that $r k\left[y, \succ_{1}\right]>$ $i^{*}+1$ and $r k\left[y, \succ_{l}\right] \leq i^{*}$ for some $l \in\left\{p+1, \ldots, n_{j}\right\}$. Now pick two alternatives $u, v \in X_{j} \backslash Y$ and a preference relation $\succ_{k}, k \in\{2, \ldots, p\}$, such that $r k\left[u, \succ_{1}\right]=$ $r k\left[v, \succ_{k}\right]=i^{*}+1$. If $u \succ_{k} y$ or $v \succ_{1} y$, then the pair $\{u, y\}$ or the pair $\{v, y\}$, respectively, violates ERD, since $y \succ_{l} u$ and $y \succ_{l} v$ by A.4. Similarly, it can be verified that if $v \succ_{k} y \succ_{k} u$ and $u \succ_{1} y \succ_{1} v$, then at least one of the pairs $\{u, v\}$, $\{v, y\}$ or $\{u, y\}$ violate ERD by A.4. We have thus derived a contradiction, hence A.5 holds. Together with A.4, this implies that all alternatives ranked below the $i^{*}$ th position in the first $p$ orderings must also be ranked below the $i^{*}$ th position in all remaining orderings. But this means again that $T_{i^{*}}\left(\succ_{1}\right)=\ldots=T_{i^{*}}\left(\succ_{n_{j}}\right)$, contradicting the definition of $X_{j}$. Thus, we must have at least three different top alternatives in $\mathcal{P}_{j}$.

Step 3: We now show that any three top alternatives in $\mathcal{P}_{j}$ produce a Condorcet cycle. Pick preferences $\succ_{k}, \succ_{m}, \succ_{l} \in \mathcal{P}_{j}$ having the three different alternatives $a, b, c \in$ $X_{j}$, respectively, on top. Without loss of generality we may assume that $a \succ_{k} b \succ_{k} c$. Now it can easily be verified that from the four possibilites

$$
\begin{aligned}
& {\left[a \succ_{k} b \succ_{k} c, b \succ_{m} a \succ_{m} c, c \succ_{l} a \succ_{l} b\right],} \\
& {\left[a \succ_{k} b \succ_{k} c, b \succ_{m} c \succ_{m} a, c \succ_{l} a \succ_{l} b\right],} \\
& {\left[a \succ_{k} b \succ_{k} c, b \succ_{m} a \succ_{m} c, c \succ_{l} b \succ_{l} a\right], \text { and }} \\
& {\left[a \succ_{k} b \succ_{k} c, b \succ_{m} c \succ_{m} a, c \succ_{l} b \succ_{l} a\right]}
\end{aligned}
$$

only the Condorcet cycle satisfies ERD.

Step 4: We claim that for any three different top alternatives $a, c$ and $b$, where $r k\left[a, \succ_{k}\right]=r k\left[c, \succ_{l}\right]=r k\left[b, \succ_{m}\right]=1$, there exists $t_{k, l, m} \in\left\{1, \ldots, q_{j}\right\}$ such that $\left\{\left.\succ_{l}\right|_{\left[1, t_{k, l, m}\right]},\left.\succ_{m}\right|_{\left[1, t_{k, l, m}\right]}\right\} \subseteq \mathcal{Z}\left(\left.\succ_{k}\right|_{\left[1, t_{k, l, m}\right]}\right)$. By Step 3 we can assume that the top elements are ordered in the following way $a \succ_{k} b \succ_{k} c, c \succ_{l} a \succ_{l} b, b \succ_{m} c \succ_{m} a$. Take an alternative $x$ such that $c \succ_{l} x \succ_{l} a$. Suppose that $a \succ_{m} x$; this implies $c \succ_{k} x$ by ERD. But then ERD must be violated, since $x$ cannot maintain its rank difference to both $a$ and $b$ in $\succ_{k}$ as well as in $\succ_{m}$. Hence, we have $x \succ_{m} a$, and by ERD, the rank difference between $x$ and $a$ has to be the same in $\succ_{l}$ as in $\succ_{m}$. In a similar way one can establish that $r k\left[b, \succ_{k}\right]-r k\left[z, \succ_{k}\right]=r k\left[b, \succ_{l}\right]-r k\left[z, \succ_{l}\right]$ for any $a \succ_{k} z \succ_{k} b$ and that $r k\left[c, \succ_{m}\right]-r k\left[y, \succ_{m}\right]=r k\left[c, \succ_{k}\right]-r k\left[y, \succ_{k}\right]$ for any $b \succ_{m} y \succ_{m} c$.

Next we pick an alternative $z$ satisfying $a \succ_{l} z \succ_{l} b$. Then $z$ must be ranked below $a$ in $\succ_{m}$, since by the above argument, $\left\{w: c \succ_{l} w \succ_{l} a\right\}=\left\{w: c \succ_{m} w \succ_{m} a\right\}$, $\left\{w: b \succ_{m} w \succ_{m} c\right\}=\left\{w: b \succ_{k} w \succ_{k} c\right\}$, and $\left\{w: a \succ_{k} w \succ_{k} b\right\}=\left\{w: a \succ_{l}\right.$ $\left.w \succ_{l} b\right\}$. Hence, by ERD, $r k\left[c, \succ_{l}\right]-r k\left[z, \succ_{l}\right]=r k\left[c, \succ_{m}\right]-r k\left[z, \succ_{m}\right]$. Similarly, $r k\left[a, \succ_{k}\right]-r k\left[y, \succ_{k}\right]=r k\left[a, \succ_{l}\right]-r k\left[y, \succ_{l}\right]$ for any $b \succ_{k} y \succ_{k} c$, and $r k\left[b, \succ_{m}\right]-$ $r k\left[x, \succ_{m}\right]=r k\left[b, \succ_{k}\right]-r k\left[x, \succ_{k}\right]$ for any $c \succ_{m} x \succ_{m} a$. Finally, observe that we can choose $t_{k, l, m}=r k\left[b, \succ_{k}\right]-r k\left[a, \succ_{k}\right]+r k\left[a, \succ_{l}\right]-r k\left[c, \succ_{l}\right]+r k\left[c, \succ_{m}\right]-r k\left[b, \succ_{m}\right]$.

\footnotetext{
${ }^{5}$ Observe that this implies $r k\left[b, \succ_{1}\right] \leq i^{*}$, since $b \in Y$ and $r k\left[b, \succ_{p+1}\right]=1$.
} 
Step 5: Now we can complete the proof. Assume that $a, b, c, d_{4}, \ldots d_{n_{j}}$ are the top alternatives of $\succ_{1}, \ldots, \succ_{n_{j}}$, respectively, where $a, b$ and $c$ are pairwise distinct. Apply Step 4 to preferences $\succ_{1}, \succ_{2}$ and $\succ_{3}$, and pick another preference relation $\succ_{m} \in \mathcal{P}_{j}$ arbitrarily.

First, if one of the three first top alternatives, say $c$, is also the top alternative of $\succ_{m}$, then Step 3 and ERD imply $r k\left[a, \succ_{3}\right]-r k\left[c, \succ_{3}\right]=r k\left[a, \succ_{m}\right]-r k\left[c, \succ_{m}\right]$ and $r k\left[b, \succ_{3}\right]-r k\left[c, \succ_{3}\right]=r k\left[b, \succ_{m}\right]-r k\left[c, \succ_{m}\right]$. Hence, by Step 4 we must have $\left.\succ_{3}\right|_{\left[1, t_{1,2,3}\right]}=\left.\succ_{m}\right|_{\left[1, t_{1,2,3}\right]} \in \mathcal{Z}\left(\left.\succ_{1}\right|_{\left[1, t_{1,2,3}\right]}\right)$.

Second, suppose that $d_{m} \in X_{j}$ is distinct from $a, b$ and $c$. Then it can be easily verified that $\left.\succ_{2}\right|_{\left[1, t_{1,2,3}\right]} \in \mathcal{Z}\left(\left.\succ_{1}\right|_{\left[1, t_{1,2,3}\right]}\right)$ and $\left.\succ_{2}\right|_{\left[1, t_{1,2, m}\right]} \in \mathcal{Z}\left(\left.\succ_{1}\right|_{\left[1, t_{1,2, m}\right]}\right)$ implies $t_{1,2,3}=t_{1,2, m}$.

Thus, in both cases we obtain $T_{t_{1,2,3}}\left(\succ_{1}\right)=\ldots=T_{t_{1,2,3}}\left(\succ_{n_{j}}\right)$. Therefore, we must have $t_{1,2, m}=q_{j}$ for all $m \in\left\{3, \ldots, n_{j}\right\}$ by the construction of $X_{j}$. This completes the proof of Proposition 1.

For the proof of Theorem 3, we need the following series of lemmas. Given a profile of preferences $\left(\succ_{1}, \ldots, \succ_{n}\right) \in \mathcal{P}^{n}$, we say that alternatives $A \subseteq X$ are indifferent on the top if

$$
\sum_{i=1}^{n} r k\left[a, \succ_{i}\right]=\sum_{i=1}^{n} r k\left[b, \succ_{i}\right]<\sum_{i=1}^{n} r k\left[c, \succ_{i}\right]
$$

for all $a, b \in A$ and all $c \in X \backslash A$.

Lemma A.1 If there exists a preference profile $\left(\succ_{1}, \ldots, \succ_{n}\right) \in \mathcal{P}^{n}$ with alternatives $\{x, y\} \subseteq X$ being indifferent on the top and violating ERD, then there exists a tiebreaking rule such that Borda count is manipulable on $\mathcal{P}$.

Proof of Lemma A.1 Suppose that profile $\Pi:=\left(\succ_{1}, \ldots, \succ_{n}\right) \in \mathcal{P}^{n}$ has alternatives $x$ and $y$ violating ERD indifferent on the top. If according to $\Pi$ we have

$$
\sum_{i=1}^{n} r k\left[x, \succ_{i}\right]=\sum_{i=1}^{n} r k\left[y, \succ_{i}\right] \geq \sum_{i=1}^{n} r k\left[c, \succ_{i}\right]-2(\# X-1)
$$

for some $c \in X \backslash\{x, y\}$, then we can take a 'multiple' of profile $\Pi$ consisting of $l$ preferences of type $\succ_{i}$ for each $i$ such that

$$
l \sum_{i=1}^{n} r k\left[x, \succ_{i}\right]=l \sum_{i=1}^{n} r k\left[y, \succ_{i}\right]<l \sum_{i=1}^{n} r k\left[c, \succ_{i}\right]-2(\# X-1)
$$

for all $c \in X \backslash\{x, y\}$ and $l$ sufficiently large by (A.6). This ensures that if only one voter reveals another preference relation, then either $x$ or $y$ will still be the Borda winning alternative. For notational convenience we will assume in what follows that $\Pi=\left(\succ_{1}, \ldots, \succ_{n}\right)$ already satisfies (A.7).

Since $x$ and $y$ are indifferent on the top, profile $\Pi$ must have voters with preferences $\succ_{i}$ and $\succ_{j}$ such that $x \succ_{i} y$ and $y \succ_{j} x$. Suppose that there exists another preference $\succ^{\prime} \in \mathcal{P}$ such that $x \succ^{\prime} y$ and $r k\left[y, \succ^{\prime}\right]-r k\left[x, \succ^{\prime}\right] \neq r k\left[y, \succ_{i}\right]-r k\left[x, \succ_{i}\right]$. Now if $r k\left[y, \succ^{\prime}\right]-r k\left[x, \succ^{\prime}\right]>r k\left[y, \succ_{i}\right]-r k\left[x, \succ_{i}\right]$, then, taking a tie-breaking rule selecting $y$ as the winner in case of ties between $x$ and $y$, a voter having preference $\succ_{i}$ could manipulate by revealing preference $\succ^{\prime}$. Otherwise, if $r k\left[y, \succ^{\prime}\right]-r k\left[x, \succ^{\prime}\right]<r k\left[y, \succ_{i}\right]-$ 
$r k\left[x, \succ_{i}\right]$, then we take the tie-breaking rule, which selects $x$ as the winner in case of ties between $x$ and $y$. Consider profile $\left(\succ_{1}, \ldots, \succ_{i-1}, \succ^{\prime}, \succ_{i+1}, \ldots, \succ_{n}\right)$, which has $y$ as the Borda winner. Clearly, voter $i$ can achieve a tie between $x$ and $y$ by revealing $\succ_{i}$ instead of $\succ^{\prime}$ and therefore, enforce that $x$ will be chosen, which he prefers to $y$.

Finally, if there does not exist a preference $\succ^{\prime} \in \mathcal{P}$ such that $x \succ^{\prime} y$ and $r k\left[y, \succ^{\prime}\right]-$ $r k\left[x, \succ^{\prime}\right] \neq r k\left[y, \succ_{i}\right]-r k\left[x, \succ_{i}\right]$, then there exists a preference $\succ^{\prime} \in \mathcal{P}$ such that $y \succ^{\prime} x$ and $r k\left[x, \succ^{\prime}\right]-r k\left[y, \succ^{\prime}\right] \neq r k\left[x, \succ_{i}\right]-r k\left[y, \succ_{i}\right]$, since $x$ and $y$ violate ERD. Hence, to complete the proof we just have to exchange the roles of $x$ and $y$ while repeating the arguments of the previous paragraph.

The next lemma is a simple corollary to Lemma A.1.

Lemma A.2 If in a rich domain $\mathcal{P}$ there exists a preference $\succ$ with its top two alternatives violating ERD, then there exists a tie-breaking rule such that Borda count is manipulable on $\mathcal{P}$.

Proof of Lemma A.2 Let $r k[x, \succ]=1$ and $r k[y, \succ]=2$. Since $\mathcal{P}$ is a rich domain, we can find a preference $\succ^{\prime} \in \mathcal{P}$, which has $y$ as the top alternative. We define $d:=$ $r k\left[x, \succ^{\prime}\right]-r k\left[y, \succ^{\prime}\right]$. Now taking one voter with $\succ^{\prime}$ and $d$ voters with $\succ$ we obtain a profile that has $\{x, y\}$ indifferent on the top, since $y$ dominates any $z \in X \backslash\{x, y\}$. Now apply Lemma A.1.

Sometimes the set of alternatives that are indifferent on the top will contain more than two alternatives. In this case the following lemma turns out to be helpful in many cases.

Lemma A.3 Suppose that $\mathcal{P}$ is a rich domain. If there exist two distinct preferences $\succ, \succ^{\prime} \in \mathcal{P}$ and an alternative $y \in X$ satisfying

- $r k[y, \succ] \geq 2$

- $\forall x \in X: x \succ y \Rightarrow x \succ^{\prime} y$,

- $\forall x \in X: x \succ y \Rightarrow r k[y, \succ]-r k[x, \succ] \neq r k\left[y, \succ^{\prime}\right]-r k\left[x, \succ^{\prime}\right]$,

then there exists a tie-breaking rule such that Borda count is manipulable on $\mathcal{P}$.

Proof of Lemma A.3 Let $k=r k[y, \succ]$. For all $i \in\{1, \ldots, k-1\}$ we shall denote by $x_{i}$ the alternative with $r k\left[x_{i}, \succ\right]=i$. Pick a preference $\succ^{\prime \prime} \in \mathcal{P}$ having $y$ as the top alternative. We define values $d_{i}:=r k\left[x_{i}, \succ^{\prime \prime}\right]-r k\left[y, \succ^{\prime \prime}\right]$ for all $i \in\{1, \ldots, k-1\}$. Clearly, we have $r k[y, \succ]-r k\left[x_{i}, \succ\right]=k-i$ for all $i \in\{1, \ldots, k-1\}$. Now let $J:=\arg \min _{i \in\{1, \ldots, k-1\}} \frac{d_{i}}{k-i}$ and $A:=\left\{x_{j} \in X: j \in J\right\}$. Pick an arbitrary $j \in J$. Then it can be verified that a profile consisting of $d_{j}$ preferences of type $\succ$ and $k-j$ preferences of type $\succ^{\prime \prime}$ makes alternatives $\{y\} \cup A$ indifferent on the top. In particular, we will take a profile $\left(\succ_{i}\right)_{i=1}^{n} \in \mathcal{P}^{n}$ consisting of $l d_{j}$ preferences of type $\succ$ and $l(k-j)$ preferences of type $\succ^{\prime \prime}$ for which

$$
\sum_{i=1}^{n} r k\left[y, \succ_{i}\right]=\sum_{i=1}^{n} r k\left[a, \succ_{i}\right]<\sum_{i=1}^{n} r k\left[b, \succ_{i}\right]-2(\# X-1)
$$

is satisfied for all $a \in A$ and all $b \in X \backslash(\{y\} \cup A)$, where $l$ is a suffciently large positive integer. Thus, we can restrict our attention to alternatives in $\{y\} \cup A$. 
We have to deal with two cases. First, suppose that there exists an alternative $a \in A$ such that $r k[y, \succ]-r k[a, \succ]<r k\left[y, \succ^{\prime}\right]-r k\left[a, \succ^{\prime}\right]$. If we select a tie-breaking rule, which prefers $y$ to all alternatives in $A$, then a voter having preference $\succ$ can manipulate by revealing $\succ^{\prime}$, since he prefers any alternative in $A$ to $y$.

Second, suppose that for all alternatives $a \in A$ we have $r k[y, \succ]-r k[a, \succ]>$ $r k\left[y, \succ^{\prime}\right]-r k\left[a, \succ^{\prime}\right]$. If we select a tie-breaking rule, which prefers all alternatives in $A$ to $y$, and consider a profile in which one voter's preference of type $\succ$ in $\left(\succ_{i}\right)_{i=1}^{n}$ is replaced by $\succ^{\prime}$, then this voter with preference $\succ^{\prime}$ can manipulate by revealing $\succ$, since he prefers any alternative in $A$ to $y$. This completes the proof of the lemma.

Proof of Theorem 3 We prove the contraposition of the statement, i.e., if a rich domain $\mathcal{P} \subseteq \mathcal{P}_{X}$ does not satisfy ERD, then there exists a tie-breaking rule for which the Borda count is manipulable on $\mathcal{P}$. Hence, suppose that the rich domain $\mathcal{P} \subseteq \mathcal{P}_{X}$ does not satisfy ERD.

Step 1: We can assume without loss of generality that the rich domain $\mathcal{P}$ violating ERD consists of exactly $q$ preferences (recall that $q=\# X$ ). This can be verified as follows. Take an arbitrary rich domain $\mathcal{P}$ violating ERD with $\# \mathcal{P}>q$. Choose $q$ preferences from $\mathcal{P}$ with different top alternatives, and denote the corresponding domain by $\mathcal{P}_{0}$. If $\mathcal{P}_{0}$ violates ERD, then we are done. On the other hand, if $\mathcal{P}_{0}$ does not violate ERD, then $\mathcal{P}_{0}=\mathcal{Z}(\succ)$ for any $\succ \in \mathcal{P}_{0}$ by Proposition 1. Consider any preference ordering $\succ_{0} \in \mathcal{P} \backslash \mathcal{P}_{0}$ and replace the preference in $\mathcal{P}_{0}$ with the same top alternative as $\succ_{0}$ by the ordering $\succ_{0}$. As is easily verified, the resulting domain violates ERD. Henceforth, we thus assume that $\mathcal{P}=\left\{\succ_{1}, \ldots, \succ_{q}\right\}$ is rich and violates ERD.

Step 2: We will construct a "chain" of alternatives and preferences. Start with preference $\succ_{1}$ and denote its top alternative by $x_{1}$ and its second ranked alternative by $x_{2}$. Without loss of generality we can assume that $\succ_{2}$ has $x_{2}$ on top. To describe how the procedure goes on suppose that we have already obtained a sequence of distinct alternatives $x_{1}, \ldots, x_{k}$ such that $r k\left[x_{i}, \succ_{i}\right]=1$ for all $i \in\{1, \ldots, k\}$ and $r k\left[x_{i}, \succ_{i-1}\right]=2$ for all $i \in\{2, \ldots, k\}$. Now we define $x_{k+1}$ recursively to be the second ranked alternative of $\succ_{k}$. We have found a "chain" if $x_{k+1}$ equals one of the alternatives $x_{1}, \ldots, x_{k}$. Otherwise, we can suppose without loss of generality that $x_{k+1}$ is the top alternative of $\succ_{k+1}$. We iterate the described procedure until we obtain a "chain" of alternatives. Clearly, this procedure terminates in at most $q$ steps. Thus, we can determine indices $m, p \in\{1, \ldots, q\}$ such that $m<p, x_{m}, \ldots, x_{p}$ are all distinct, $r k\left[x_{i}, \succ_{i}\right]=1$ and $r k\left[x_{i+1}, \succ_{i}\right]=2$ for all $i \in\{m, \ldots, p-1\}$, and $r k\left[x_{p}, \succ_{p}\right]=1$ and $r k\left[x_{m}, \succ_{p}\right]=2$. In what follows we can assume without loss of generality that $m=1$. Nevertheless we will still denote the length of the chain by $p$. Furthermore, let $X^{\prime}:=\left\{x_{1}, \ldots, x_{p}\right\}$ and $\mathcal{P}_{1}:=\left\{\succ_{1}, \ldots, \succ_{p}\right\}$.

Step 3: We can manipulate by Lemma A.2 for some tie-breaking rules if there exists a preference $\succ_{i} \in \mathcal{P}_{1}$ in which the top two alternatives violate ERD. Hence, in the following analysis we can assume that the top two alternatives of all $\succ_{1}, \ldots, \succ_{p}$ satisfy ERD. But this implies that the top $p$ alternatives of the preferences in $\mathcal{P}_{1}$ follow the pattern shown in Table 2. Clearly, if $p=q$, we cannot have a violation of ERD by Proposition 1, hence we must have $p<q$.

Case (i): Suppose that there exists an alternative $y \in X$ that is ranked by two distinct preferences $\succ_{i}$ and $\succ_{j}(i, j \in\{1, \ldots, p\})$ at the $p+1$ th position. Then $y$ violates ERD with all alternatives $x_{1}, \ldots, x_{p}$, since $\mathcal{P}$ is a rich domain and all alternatives $x_{1}, \ldots, x_{p}$ are ranked differently according to $\succ_{i}$ and $\succ_{j}$ while $y$ is ranked identically 
Table 2: A full cycle on the top

\begin{tabular}{llllll}
$\succ_{1}$ & $\succ_{2}$ & $\ldots$ & $\succ_{p-1}$ & $\succ_{p}$ & $\ldots$ \\
\hline$x_{1}$ & $x_{2}$ & $\ldots$ & $x_{p-1}$ & $x_{p}$ & $\ldots$ \\
$x_{2}$ & $x_{3}$ & $\ldots$ & $x_{p}$ & $x_{1}$ & $\ldots$ \\
$\vdots$ & $\vdots$ & $\ldots$ & $\vdots$ & $\vdots$ & \\
& &. & & & \\
$x_{p-1}$ & $x_{p}$ & $\ldots$ & $x_{p-3}$ & $x_{p-2}$ & $\ldots$ \\
$x_{p}$ & $x_{1}$ & $\ldots$ & $x_{p-2}$ & $x_{p-1}$ & $\ldots$ \\
$\vdots$ & $\vdots$ & & $\vdots$ & $\vdots$ &
\end{tabular}

by these two preferences. Hence, taking $\succ_{i}, \succ_{j}$ and $y$ we can apply Lemma A.3.

Case (ii): Suppose that the alternatives $y_{1}, \ldots, y_{p} \in X$ are all distinct and are ranked $p+1$ th by the preferences $\succ_{1}, \ldots, \succ_{p}$, respectively. Let $Y:=\left\{y_{1}, \ldots, y_{p}\right\}$.

We claim that if there exists an alternative $y_{i} \in Y$ and a preference $\succ_{j} \in \mathcal{P}_{1}$ such that $r k\left[y_{i}, \succ_{j}\right]-r k\left[x_{i}, \succ_{j}\right] \neq p$, then $y_{i}$ violates ERD with all alternatives in $X^{\prime}$, and manipulation is possible by Lemma A.3, taking $\succ_{i}, \succ_{j}$ and $y_{i}$ as $\succ, \succ^{\prime}$ and $y$, respectively. We check this claim without loss of generality for alternative $y_{p}{ }^{6}$ Of course, $r k\left[y_{p}, \succ_{p}\right]-r k\left[x_{p}, \succ_{p}\right]=p$ and therefore, $r k\left[y_{p}, \succ_{j}\right]-r k\left[x_{p}, \succ_{j}\right] \neq p$ implies that $x_{p}$ and $y_{p}$ violate ERD. Suppose that $d:=r k\left[y_{p}, \succ_{j}\right]-r k\left[x_{p}, \succ_{j}\right]<p$. Note that we have $r k\left[x_{p}, \succ_{j}\right]=p-j+1$ and therefore, it follows that $r k\left[y_{p}, \succ_{p}\right]-r k\left[x_{i}, \succ_{p}\right]=$ $p-i>r k\left[y_{p}, \succ_{j}\right]-r k\left[x_{i}, \succ_{j}\right]=d-i$ for all $i \in\{1, \ldots, j-1\}$. In addition, for all $i \in\{j, \ldots, p-1\}$ we have $r k\left[y_{p}, \succ_{p}\right]-r k\left[x_{i}, \succ_{p}\right]=r k\left[x_{p}, \succ_{j}\right]-r k\left[x_{i}, \succ_{j}\right]<$ $r k\left[y_{p}, \succ_{j}\right]-r k\left[x_{i}, \succ_{j}\right]$. Now suppose that $d=r k\left[y_{p}, \succ_{j}\right]-r k\left[x_{p}, \succ_{j}\right]>p$. Then clearly, $r k\left[y_{p}, \succ_{j}\right]-r k\left[x_{i}, \succ_{j}\right]>d>p>r k\left[y_{p}, \succ_{p}\right]-r k\left[x_{i}, \succ_{p}\right]=p-i$ for all $i \in\{j, \ldots, p-1\}$. Furthermore, for all $i \in\{1, \ldots, j-1\}$ we have $r k\left[y_{p}, \succ_{p}\right]-r k\left[x_{i}, \succ_{p}\right]=p-i<$ $r k\left[y_{p}, \succ_{j}\right]-r k\left[x_{i}, \succ_{j}\right]=d-i$. Hence, in any case $y_{p}$ and $x_{i}$ violate ERD for all $i \in\{1, \ldots, p\}$.

We still have to investigate the case in which for all alternatives $y_{i} \in Y$ and for all preferences $\succ_{j} \in \mathcal{P}_{1}$ we have $r k\left[y_{i}, \succ_{j}\right]-r k\left[x_{i}, \succ_{j}\right]=p$. For this case the first $p$ preferences are illustrated in Table 3 . If $2 p<q$, then we can mimic the arguments given so far for alternatives ranked, by some preference relations in $\mathcal{P}_{1}$, at the $2 p+1$ th position. By doing so, in a similar way as in case (i), we can derive that manipulation is possible through an appropriately selected tie-breaking rule if an alternative is ranked twice at the $2 p+1$ th position by some preferences in $\mathcal{P}_{1}$. Otherwise, let $z_{i}$ be the alternative for which $r k\left[z_{i}, \succ_{i}\right]=2 p+1$ and let $Z:=\left\{z_{1}, \ldots, z_{p}\right\}$. Now, in an analogous way as in the beginning part of case (ii) one can argue that we can manipulate if there exists an alternative $z_{i} \in Z$ such that there exists a preference $\succ_{j} \in \mathcal{P}_{1}$ such that $r k\left[z_{i}, \succ_{j}\right]-r k\left[y_{i}, \succ_{j}\right] \neq p$. The case that remains to be investigated whenever $3 p \leq q$ is illustrated in Table 4 .

Alternatives $y_{1}, \ldots, y_{p}$ are all top alternatives of a certain preference relation since $\mathcal{P}$ is a rich domain. We shall denote the set of these preferences by $\mathcal{P}_{2}$. Without

\footnotetext{
${ }^{6}$ If we relabel the alternatives and preferences cyclically, then the claim follows for all the other alternatives $y_{1}, \ldots, y_{p-1}$ in the same way.
} 
Table 3: Two consecutive full cycles

\begin{tabular}{llllll}
$\succ_{1}$ & $\succ_{2}$ & $\ldots$ & $\succ_{p-1}$ & $\succ_{p}$ & $\ldots$ \\
\hline$x_{1}$ & $x_{2}$ & $\ldots$ & $x_{p-1}$ & $x_{p}$ & $\ldots$ \\
$x_{2}$ & $x_{3}$ & $\ldots$ & $x_{p}$ & $x_{1}$ & $\ldots$ \\
$\vdots$ & $\vdots$ & $\ldots$ & $\vdots$ & $\vdots$ & \\
& &. & & & \\
$x_{p-1}$ & $x_{p}$ & $\ldots$ & $x_{p-3}$ & $x_{p-2}$ & $\ldots$ \\
$x_{p}$ & $x_{1}$ & $\ldots$ & $x_{p-2}$ & $x_{p-1}$ & $\ldots$ \\
$y_{1}$ & $y_{2}$ & $\ldots$ & $y_{p-1}$ & $y_{p}$ & $\ldots$ \\
$y_{2}$ & $y_{3}$ & $\ldots$ & $y_{p}$ & $y_{1}$ & $\ldots$ \\
$\vdots$ & $\vdots$ & $\ldots$ & $\vdots$ & $\vdots$ & \\
$\vdots$ & & & & & \\
$y_{p-1}$ & $y_{p}$ & $\ldots$ & $y_{p-3}$ & $y_{p-2}$ & $\ldots$ \\
$y_{p}$ & $y_{1}$ & $\ldots$ & $y_{p-2}$ & $y_{p-1}$ & $\ldots$ \\
$\vdots$ & $\vdots$ & & $\vdots$ & $\vdots$ &
\end{tabular}

Table 4: Three consecutive full cycles

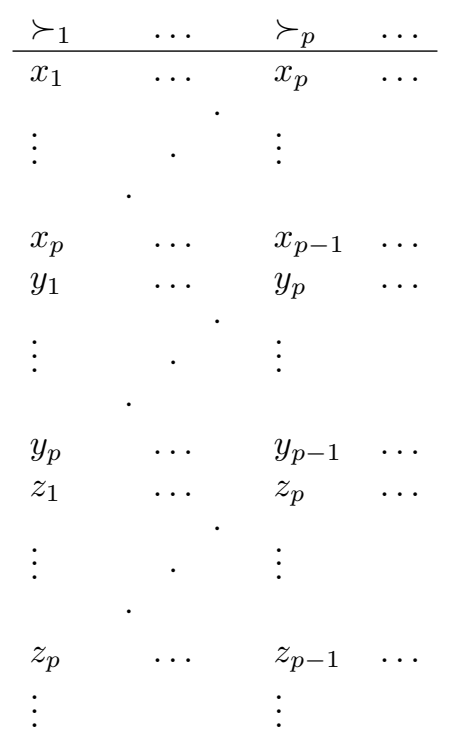


Table 5: Two full cycles on the top

\begin{tabular}{|c|c|c|c|c|c|c|}
\hline$\succ_{1}$ & $\ldots$ & $\succ_{p}$ & $\succ_{p+1}$ & $\ldots$ & $\succ_{2 p}$ & $\ldots$ \\
\hline$x_{1}$ & $\ldots$ & $x_{p}$ & $y_{1}$ & $\ldots$ & $y_{p}$ & $\ldots$ \\
\hline : & . & $\vdots$ & $\vdots$ & . & $\vdots$ & \\
\hline$x_{p}$ & $\ldots$ & $x_{p-1}$ & $y_{p}$ & $\ldots$ & $y_{p-1}$ & $\ldots$ \\
\hline$y_{1}$ & & $y_{p}$ & : & & : & \\
\hline : & $\cdot$ & : & & & & \\
\hline$y_{p}$ & $\ldots$ & $y_{p-1}$ & & & & \\
\hline$z_{1}$ & & $z_{p}$ & & & & \\
\hline : & $\cdot$ & $\vdots$ & & & & \\
\hline$z_{p}$ & $\ldots$ & $z_{p-1}$ & & & & \\
\hline$\vdots$ & & $\vdots$ & & & & \\
\hline
\end{tabular}

loss of generality we can assume that $r k\left[y_{i}, \succ_{p+i}\right]=1$ for all $i \in\{1, \ldots, p\}$. Thus, $\mathcal{P}_{2}=\left\{\succ_{p+1}, \ldots, \succ_{2 p}\right\} \subset \mathcal{P}$. In what follows we have to consider four subcases.

Subcase (a): There exists a preference $\succ_{p+i} \in \mathcal{P}_{2}$ that ranks an alternative $u \in$ $X \backslash\left(X^{\prime} \cup Y\right)$ second, i.e., $r k\left[u, \succ_{p+i}\right]=2$. Then $y_{i}$ and $u$ violate ERD and $\mathcal{P}$ is manipulable with respect to an appropriate tie-breaking rule by Lemma A.2.

Subcase (b): The set of second ranked alternatives of all preferences in $\mathcal{P}_{2}$ is a subset of $Y$. If there exists a preference in $\mathcal{P}_{2}$ with top two alternatives violating ERD, then we can apply LemmaA.2. Otherwise, if the top two alternatives of all preferences in $\mathcal{P}_{2}$ satisfy ERD, then $\mathcal{P}_{2}$ must have a very special structure, since $y_{i}$ is ranked just above $y_{i \oplus 1}$ for all $i \in\{1, \ldots, p\}$ whenever $y_{i}$ is ranked above $\left.y_{i \oplus 1}\right]^{7}$ Thus, we must have preferences as shown in Table 5 . Let

$$
A:=\left\{x \in X^{\prime}: \sum_{i=1}^{p} r k\left[x, \succ_{p+i}\right] \leq \sum_{i=1}^{p} r k\left[u, \succ_{p+i}\right] \text { for all } u \in X^{\prime}\right\} .
$$

Now pick an alternative $x_{i} \in A$ and we will make $A \cup Y$ indifferent on the top. Define $d:=\left(\sum_{j=1}^{p} r k\left[x_{i}, \succ_{p+j}\right]\right)-\frac{1}{2} p(p+1)$. For each $\succ \in \mathcal{P}_{1}$ taking $d$ voters and for each $\succ^{\prime} \in \mathcal{P}_{2}$ taking $p^{2}$ voters we obtain a profile in which all $x \in A$ and all $y \in Y$ are indifferent on the top. In particular, any $x \in A$ beats all alternatives in $X^{\prime} \backslash A$ and any $y \in Y$ beats all alternatives in $X \backslash\left(X^{\prime} \cup Y\right)$, while alternatives $x \in A$ and $y \in Y$ receive the same Borda score. Pick an alternative $x_{i} \in A$ and consider a voter having preference $\succ_{i \oplus 1}$. Suppose that the tie-breaking rule prefers $y_{i \oplus 1}$ to $x_{i}$ and $x_{i}$ to all

${ }^{7}$ For two integers $k, l \in\{1, \ldots, p\}$, if $k+l \neq p$ and $k+l \neq 2 p$, we define $k \oplus l:=(k+l) \bmod p$, while if $k+l=p$ or $k+l=2 p$, we define $k \oplus l:=p$. 
Table 6: The final case of subcase (c)

\begin{tabular}{lllllll}
$\succ_{1}$ & $\succ_{2}$ & $\succ_{3}$ & $\succ_{4}$ & $\succ_{5}$ & $\succ_{6}$ & $\ldots$ \\
\hline$x_{1}$ & $x_{2}$ & $x_{3}$ & $y_{1}$ & $y_{2}$ & $y_{3}$ & $\ldots$ \\
$x_{2}$ & $x_{3}$ & $x_{1}$ & $x_{1}$ & $x_{2}$ & $x_{3}$ & $\ldots$ \\
$x_{3}$ & $x_{1}$ & $x_{2}$ & $x_{2}$ & $x_{3}$ & $x_{1}$ & $\ldots$ \\
$y_{1}$ & $y_{2}$ & $y_{3}$ & $x_{3}$ & $x_{1}$ & $x_{2}$ & $\ldots$ \\
$y_{2}$ & $y_{3}$ & $y_{1}$ &. &. &. & $\ldots$ \\
$y_{3}$ & $y_{1}$ & $y_{2}$ & $y_{2}$ & $y_{3}$ & $y_{1}$ & $\ldots$ \\
$z_{1}$ & $z_{2}$ & $z_{3}$ & $y_{3}$ & $y_{1}$ & $y_{2}$ & $\ldots$ \\
$z_{2}$ & $z_{3}$ & $z_{1}$ & $\cdot$ & $\cdot$ & $\cdot$ & \\
$z_{3}$ & $z_{1}$ & $z_{2}$ &. &. &. & \\
$\vdots$ & $\vdots$ & $\vdots$ & $\vdots$ & $\vdots$ & $\vdots$ &
\end{tabular}

other alternatives. Then a voter having preference $\succ_{i \oplus 1}$ could manipulate by revealing $\succ_{i \oplus 2 \text {. }}$

Subcase (c): The set of second ranked alternatives of all preferences in $\mathcal{P}_{2}$ is a subset of $X^{\prime}$. If there exists a preference $\succ_{p+j} \in \mathcal{P}_{2}$ that ranks an alternative $x_{i}$ with $i \neq j$ second, then the top two alternatives of $\succ_{p+j}$ violate $\mathrm{ERD}^{8}$ and therefore, by Lemma A.2 we can find a tie-breaking rule making manipulation possible. Hence, in what follows we can assume that $r k\left[x_{i}, \succ_{p+i}\right]=2$ for all $i \in\{1, \ldots, p\}$. Since we know that the alternatives in $X^{\prime}$ satisfy ERD, $\mathcal{P}_{2}$ must have again a very special structure because $x_{i}$ is ranked just above $x_{i \oplus 1}$ for all $i \in\{1, \ldots, p\}$ whenever $x_{i}$ is ranked above $x_{i \oplus 1}$. Thus, any $\succ \in \mathcal{P}_{2}$ must rank the alternatives of $X^{\prime}$ from the 2 nd to the $p+1$ th position in a cyclic pattern. Therefore, in any preference $\succ_{p+i} \in \mathcal{P}_{2}$ we have $x_{j} \succ_{p+i} y_{j}$ for all $j \in\{1, \ldots, p\} \backslash\{i\}$. Hence, if there exists a preference $\succ_{p+i} \in \mathcal{P}_{2}$ and a pair of alternatives $x_{j}, y_{j}(j \in\{1, \ldots, p\} \backslash\{i\})$ such that $r k\left[y_{j}, \succ_{p+i}\right]-r k\left[x_{j}, \succ_{p+i}\right] \neq p$, then the top two alternatives $y_{j}$ and $x_{j}$ of $\succ_{p+j}$ violate ERD and we are done by applying Lemma A.2.

We still have to investigate the case in which for all preferences $\succ_{p+i} \in \mathcal{P}_{2}$ and for all pairs of alternatives $x_{j}, y_{j}(j \in\{1, \ldots, p\} \backslash\{i\})$ we have $r k\left[y_{j}, \succ_{p+i}\right]-r k\left[x_{j}, \succ_{p+i}\right]=p$. For the case of $p=3$ we illustrate this case in Table 6. Clearly, this case can only occur whenever $2 p<q$. Observe that the $p+2$ nd positions of each preference in $\mathcal{P}_{2}$ have to be filled with an alternative from $X \backslash\left(X^{\prime} \cup Y\right)$. Suppose that we have $r k\left[u, \succ_{p+1}\right]=p+2$ for an alternative $u \in X \backslash\left(X^{\prime} \cup Y\right)$. Then $u$ violates ERD with all alternatives ranked by $\succ_{p+1}$ above $u$ (i.e., with all alternatives in $X^{\prime} \cup\left\{y_{1}\right\}$ ), since $\mathcal{P}$ is a rich domain. More specifically, if $u \neq z_{2}$, then we can apply Lemma A.3 with $\succ_{p+1}, \succ_{1}$ and $u$; while if $u=z_{2}$, then we can apply Lemma A.3 with $\succ_{p+1}, \succ_{2}$ and $u$.

Subcase (d): We still have to investigate the case in which the second ranked alternatives in $\mathcal{P}_{2}$ come from both $X^{\prime}$ and $Y$. First, observe that as in subcase (c), if there exists a preference $\succ_{p+j} \in \mathcal{P}_{2}$ that ranks an alternative $x_{i}$ with $i \neq j$ 2nd, then $x_{i}$ and

\footnotetext{
${ }^{8}$ In fact, looking at Table 3 it is easy to verify that all pairs $x_{i}$ and $y_{j}(i \neq j)$ violate ERD, while all pairs $x_{i} \in X^{\prime}$ and $y_{i} \in Y$ satisfy ERD on $\mathcal{P}_{1}$. For instance, if $i<j$, then the sequence $\left(r k\left[x_{i}, \succ_{k}\right]\right)_{k=1}^{p}$ decreases until $k=i$ and jumps up by $p-1$ afterwards, whereas $\left(r k\left[y_{i}, \succ_{k}\right]\right)_{k=1}^{p}$ still decreases after $k=i$. Hence, the rank differences between $x_{i}$ and $y_{j}$ differ according to preferences $\succ_{i}$ and $\succ_{i+1}$ One can argue analogously in case of $1 \leq j<i \leq p$.
} 
Table 7: The final case of subcase (d)

\begin{tabular}{lllllll}
$\succ_{1}$ & $\succ_{2}$ & $\succ_{3}$ & $\succ_{4}$ & $\succ_{5}$ & $\succ_{6}$ & $\ldots$ \\
\hline$x_{1}$ & $x_{2}$ & $x_{3}$ & $y_{1}$ & $y_{2}$ & $y_{3}$ & $\ldots$ \\
$x_{2}$ & $x_{3}$ & $x_{1}$ & $y_{2}$ & $x_{2}$ &. & $\ldots$ \\
$x_{3}$ & $x_{1}$ & $x_{2}$ & $x_{2}$ & $x_{3}$ &. & $\ldots$ \\
$y_{1}$ & $y_{2}$ & $y_{3}$ & $x_{3}$ & $x_{1}$ &. & $\ldots$ \\
$y_{2}$ & $y_{3}$ & $y_{1}$ & $x_{1}$ &. &. & $\ldots$ \\
$y_{3}$ & $y_{1}$ & $y_{2}$ &. &. &. & $\ldots$ \\
$\vdots$ & $\vdots$ & $\vdots$ & $\vdots$ & $\vdots$ & $\vdots$ &
\end{tabular}

$y_{j}$ violate ERD and we can apply Lemma A.2. Hence, in what follows we can assume that if $r k\left[u, \succ_{p+i}\right]=2$ and $u \in X^{\prime}$, then $u=x_{i}$.

Second, if there exists a preference $\succ_{p+i} \in \mathcal{P}_{2}$ that ranks an alternative $y \in Y \backslash\left\{y_{i \oplus 1}\right\}$ second, then $y_{i}$ and $y$ violate ERD and we are done by Lemma A.2.

Finally, we can assume that there exists $\succ_{p+i} \in \mathcal{P}_{2}$ such that $r k\left[y_{i \oplus 1}, \succ_{p+i}\right]=2$ and $r k\left[x_{i \oplus 1}, \succ_{p+(i \oplus 1)}\right]=2$. Now if $r k\left[x_{i \oplus 1}, \succ_{p+i}\right]>3$, then the top two alternatives $y_{i \oplus 1}$ and $x_{i \oplus 1}$ of $\succ_{p+(i \oplus 1)}$ violate ERD and we are finished by Lemma A.2. Otherwise, if $r k\left[x_{i \oplus 1}, \succ_{p+i}\right]=3$, then we can make $x_{i \oplus 1}$ and $y_{i}$ indifferent on the top by taking for all preferences $\succ \in \mathcal{P}_{1}$ two voters each and $p^{2}$ voters with $\succ_{p+i}{ }^{9}$ In particular, $y_{i}$ beats any other alternative in $X \backslash X^{\prime}$ and $x_{i \oplus 1}$ beats any other alternative in $X^{\prime}$, while $y_{i}$ and $x_{i \oplus 1}$ receive the same Borda score. Since $x_{i \oplus 1}$ and $y_{i}$ violate ERD, we can apply Lemma A.1.

\footnotetext{
${ }^{9}$ For $p=3$ and $i=1$ this case is illustrated in Table 7
} 


\section{References}

[1] Aleskerov, F. and E. Kurbanov (1999), Degree of Manipulability of Social Choice Procedures, in: A. Alkan et.al. (eds.), Current Trends in Economics, Springer, Berlin.

[2] Barberá, S., F. Gul and E. Stacchetti (1993), Generalized Median Voter Schemes and Committees, Journal of Economic Theory 61, 262-289.

[3] Barberá, S., H. Sonnenschein and L. Zhou (1991), Voting by Committees, Econometrica 59, 595-609.

[4] Black, D. (1958), The Theory of Committees and Elections, Cambridge University Press, Cambridge.

[5] Dasgupta, P. and E. Maskin (2000), On the Robustness of Majority Rule, mimeo.

[6] Favardin, P., D. Lepelley and J. Serais (2002), Borda Rule, Copeland Method and Strategic Manipulation, Review of Economic Design 7, 213-228.

[7] Gaertner, W. (2002), Domain Conditions in Social Choice Theory, Cambridge University Press, Cambridge.

[8] Maskin, E. (1995), Majority Rule, Social Welfare Functions, and Game Forms, in: K. Basu, P.K. Pattanaik and K. Suzumura (eds.), Choice, Welfare and Development, Clarendon Press, Oxford.

[9] Moulin, H. (1980), On Strategy-Proofness and Single-Peakedness, Public Choice 35, $437-455$.

[10] Moulin, H. (1988), Axioms of Cooperative Decision Making, Cambridge University Press, Cambridge.

[11] Nehring, K. and C. Puppe (2003a), On the Structure of Strategy-Proof Social Choice, mimeo.

[12] Nehring, K. and C. Puppe (2003b), Strategy-Proof Social Choice without Dictators, mimeo.

[13] Smith, D. (1999), Manipulability Measures of Common Social Choice Functions, Social Choice and Welfare 16, 639-661. 